\title{
MULTIVARIATE CONTROL CHARTS WITH A BAYESIAN NETWORK
}

\author{
Sylvain VERRON, Teodor TIPLICA, Abdessamad KOBI \\ LASQUO/ISTIA, 62, avenue Notre Dame du Lac, 49000, Angers, France \\ sylvain.verron@univ-angers.fr, teodor.tiplica@univ-angers.fr, abdessamad.kobi@univ-angers.fr
}

\begin{abstract}
Keywords: $\quad$ SPC, Bayesian network, multivariate control charts, $T^{2}$, MEWMA.
Abstract: The purpose of this article is to present an approach allowing the fault detection of a multivariate process with a bayesian network. As a discriminant analysis is easily modeled with a bayesian network, we will show that we we can consider the multivariate $T^{2}$ and MEWMA control charts as particular cases of the discriminant analysis. So, we give the structure of the bayesian network as well as the parameters of the network in order to detect faults in the multivariate space in the same manners as if we used multivariate control charts. The resulting bayesian network, with a computed threshold, is similar to the multivariate control charts.
\end{abstract}

\section{INTRODUCTION}

Nowadays, process control (or process monitoring) is becoming an essential task. Indeed, processes are increasingly complex and automatized (containing a lot of sensors and actuators). As a consequence, the control of these processes is more and more difficult. In (Chiang et al., 2001), authors give two principal approaches to perform the process control, namely, data-driven techniques and analytical techniques. The analytical technique is, in theory, the better approach. It is based on analytical (physical) models of the system and permits to simulate the system. Though, at each instant, the theoretical value of each sensor can be known for the normal operating state of the system. As a consequence, it is relatively easy to see if the real process values are similar to the theoretical values. But, the major drawback of this approach is the fact that it requires detailed models of the process. An effective detailed model can be very difficult, time consuming and expensive to obtain, particularly for large-scale systems with many variables. The data-driven approaches are a family of different techniques based on the analysis of the real data extracted from the process. These methods are based on rigorous statistical development of the process data. We can principally cite control charts, methods based on Principal Component Analysis (PCA), Projection to Latent Structure (PLS) or Discriminant Analysis (DA) (Chiang et al., 2001). The process control can be viewed as a three-step procedure: the first one is the fault detection that concludes on the presence of a disturbance in the process; secondly, it is needed to diagnosis the disturbance (fault diagnosis); finally, the process must return in normal operation state (process recovery).

Many data-driven techniques for the fault detection can be found in the literature: univariate statistical process control (Shewhart charts) (Shewhart, 1931), multivariate statistical process control ( $T^{2}$,Q MEWMA, MCUSUM charts) (Hotelling, 1947; Lowry et al., 1992; Pignatiello and Runger, 1990), and some PCA (Principal Component Analysis) based techniques (Jackson, 1985) like Moving PCA (Bakshi, 1998). In (Kano et al., 2002), authors make comparisons between these different techniques. Other important approaches are PLS (Projection to Latent Structures) based approaches (MacGregor and Kourti, 1995).

These fault detection techniques are able to detect a fault (a disturbance) in a multivariate process. The fault can be a shift of the mean affecting one or more variables. For example, it can be a step or a trend. The major drawback of these techniques is that they do not give any indication about the root cause of a detected fault, and so they are not fully exploitable in 
the industry. In order to accomplish the fault diagnosis, many approaches have been proposed (Kourti and MacGregor, 1996). The fault diagnosis procedure can also be seen as a classification task. Indeed today's processes give many measurements that can be stored in a database when the process is in control, but also in case of identified out-of-control states. Many classifiers have been developed. We can cite discriminant analysis like FDA (Fisher Discriminant Analysis) (Duda et al., 2001), SVM (Support Vector Machine) (Vapnik, 1995), kNN (k-nearest neighborhood) (Cover and Hart, 1967), ANN (Artificial Neural Networks) (Duda et al., 2001) and bayesian classifiers (Friedman et al., 1997). The performances of these classifiers are reduced in the space described by all the variable of the process. So, before the classification, a feature selection is often required in order to obtain better performances.

We can see that the methods of detection and diagnosis are numerous. Moreover, all these methods are heterogeneous. But, as their final goal is the same (process recovery), they are complementary. So, it will be of interest to have the possibility to detect and to diagnose a fault with a single tool. An interesting approach for the diagnosis can be the use of Bayesian Networks (BN) (Friedman et al., 1997). In this article, we will study a possibility to detect a fault in a multivariate process by modelling the multivariate control chart with a BN. So, detection and diagnosis of a fault would be possible on a same tool: a bayesian network.

The article is structured as follows: in the second section, we will present the utilization of the multivariate control charts for the detection of faults in a multivariate process; the third section presents some aspects on bayesian networks and particularly on bayesian network classifiers; the fourth section gives the procedure to model some multivariate control charts ( $T^{2}$ and MEWMA), with a bayesian network. In the last section, we conclude on the proposed approach and give some outlooks.

\section{MULTIVARIATE CONTROL CHARTS}

\subsection{The Hotelling $T^{2}$ control chart}

The first work in the field of fault detection in multivariate processes began in 1947 with Hotelling (Hotelling, 1947). He was the first to propose a multivariate control chart based on a statistical distance. For a process with $p$ variables, we can write the statistic $T^{2}$ as:

$$
T^{2}=(x-\mu)^{T} \Sigma^{-1}(x-\mu)
$$

where: $x$ is the observation vector of size $1 \times p$, $\mu$ is the mean vector of size $1 \times p, \Sigma$ is the variancecovariance matrix of size $p \times p$ and the symbol ${ }^{T}$ represents the transpose of a vector or a matrix.

As we can see in the equation 1 , the statistic $T^{2}$ is a scalar. So, we can plot the value of the $T^{2}$ for different time instants, and with an appropriate control limit (computed by taking into account statistical considerations), the $T^{2}$ control chart is obtained. On this chart, each point represents the information extracted form all the $p$ variables. A fault is detected when a point is beyond the control limit.

As for the univariate Shewhart control chart, the set up of the $T^{2}$ chart is made in two phases. During the first phase, parameters of the process $(\mu$ and $\Sigma$ ) are estimated. Concerning the computation of these parameters estimations and the computation of the control limit, readers can refer to the book of Montgomery (Montgomery, 1997). Once the parameters are estimated, the $T^{2}$ control chart can be drawn. It is very important to verify that the process is in control during the first phase. The second phase represents the real monitoring of the process on the assumption of a multivariate normal distribution.

\subsection{The MEWMA control chart}

As in the case of the univariate Shewhart control chart, the major drawback of the $T^{2}$ control chart is his moderate performances to detect small mean shifts. In order to solve this problem, other multivariate control charts have been proposed: MEWMA (Multivariate Exponentially Weighted Moving Average) (Lowry et al., 1992) and MCUSUM (Multivariate CUmulative SUM) (Pignatiello and Runger, 1990). These charts are respectively the multivariate analogous of the EWMA and CUSUM control charts. The principle of the MEWMA control chart is to take into account the process evolution in weighting past observations extracted from the process, as indicated in the equation 2 :

$$
y_{t}=\lambda x_{t}+(I-\lambda) y_{t-1}
$$

where $\lambda$ is a $p \times p$ diagonal weighting matrix, $I$ is the $p \times p$ identity matrix, $x_{t}$ is the observation vector $($ size $1 \times p)$ at instant $t, y_{0}=\mu$ is the mean vector (size $1 \times p$ ) of the $p$ variables.

Based on the same principle than a $T^{2}$ control chart, one can monitor the process with the statistic given in the equation 3 :

$$
T_{t}^{2}=y_{t}^{T} \Sigma_{y}^{-1} y_{t}
$$


where $y_{t}$ is the transformed observation vector at instant $t, \Sigma_{y}^{-1}$ is the inverse of the variance-covariance matrix of $y_{t}$. It can be concluded that the process is out of control as soon as the $T_{t}^{2}$ crosses the control limit. Bodden (Bodden and Rigdon, 1999) proposed an algorithm to find the control limit in order to respect a given number of false alarm and a given $\lambda$.

These multivariate control charts $\left(T^{2}\right.$ and MEWMA) are efficient to detect a fault in a multivariate process. But, as we have already said, the fault diagnosis cannot be made in the same time. So, it seems to be an interesting approach to do the fault detection and the fault diagnosis of a multivariate process by using a single tool. Several works demonstrated that bayesian networks are able to diagnose correctly the fault of a multivariate process. So, it is of interest to study if a bayesian network can accomplish the fault detection in an efficient way.

\section{BAYESIAN NETWORK CLASSIFIERS}

A Bayesian Network (BN) (Jensen, 1996; Pearl, 1988 ) is an acyclic graph where each variable is a node (that can be continuous or discrete). Edges of the graph represent dependence between linked nodes. A formal definition is given here:

A bayesian network is a triplet $\{\mathbf{G}, \mathbf{E}, \mathbf{D}\}$ where:

$\{\mathbf{G}\}$ is a directed acyclic graph, $\mathbf{G}=(V, A)$, with $V$ the ensemble of nodes of $\mathbf{G}$, and $A$ the ensemble of edges of $\mathbf{G}$,

$\{\mathbf{E}\}$ is a finite probabilistic space $(\Omega, Z, p)$, with $\Omega$ a non-empty space, $Z$ a collection of subspace of $\Omega$, and $p$ a probability measure on $Z$ with $p(\Omega)=1$,

$\{\mathbf{D}\}$ is an ensemble of random variables associated to the nodes of $\mathbf{G}$ and defined on $\mathbf{E}$ such as:

$$
p\left(V_{1}, V_{2}, \ldots, V_{n}\right)=\prod_{i=1}^{n} p\left(V_{i} \mid C\left(V_{i}\right)\right)
$$

with $C\left(V_{i}\right)$ the ensemble of causes (parents) of $V_{i}$ in the graph $\mathbf{G}$.

Bayesian network classifiers are particular $\mathrm{BN}$ (Friedman et al., 1997). They always have a discrete node $C$ coding the $k$ different classes of the system. Thus, other variables $X_{1}, \ldots, X_{p}$ represent the $p$ descriptors (variables) of the system.

A famous bayesian classifier is the Naïve Bayesian Network (NBN), also named Bayes classifier (Langley et al., 1992). This bayesian classifier makes the strong assumption that the descriptors of the system are class conditionally independent. Assuming the hypothesis of normality of each descriptor, the NBN is equivalent to the classification rule of the diagonal quadratic discriminant analysis. But, in practice, this assumption of independence and non correlated variables is not realistic. In order to deal with correlated variables, several approaches have been developed. We can cite the Tree Augmented Naïve bayesian networks (TAN) (Friedman et al., 1997). These BNs are based on a NBN but a tree is added between the descriptors. An other interesting approach is the Kononenko one (Kononenko, 1991), which represent some variables in one node. As in (Perez et al., 2006) the assumption we will make is that this variable follows a normal multivariate distribution (conditionally to the class) and we will refer to this kind of BN as Condensed Semi Naïve Bayesian Network (CSNBN).

a)

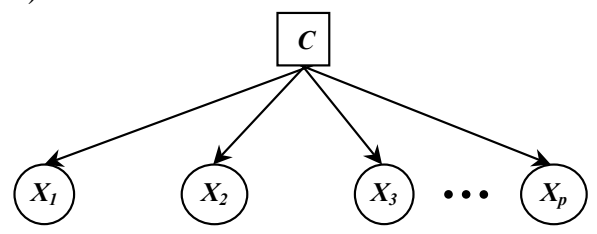

b)

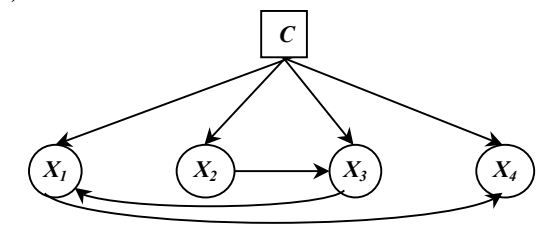

c)

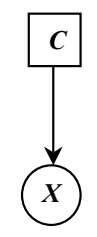

Figure 1: Different bayesian network classifiers: NBN (a), TAN (b) and CSNBN (c).

\section{MULTIVARIATE CONTROL CHARTS MODELIZED WITH BAYESIAN NETWORK}

The purpose of this article is to present a method allowing the fault detection in a multivariate process with the use of a BN. In the section 2, we have presented the multivariate control charts $T^{2}$ and MEWMA. It can be remarked that for these two charts, one needs to compute a statistical distance on 
the form of $X^{T} \Sigma^{-1} X$ which is known as the square Mahalanobis distance. This distance is also used in a Discriminant Analysis (DA).

Indeed, one can distinguish two aspects of discriminant analysis: the predictive and the classification aspect. Given a system with $p$ variables (descriptors) and with $k$ identified classes, the predictive discriminant analysis (or Fisher discriminant analysis) is generally used to find $k-1$ new descriptors of a system. These new $k-1$ descriptors (which are a linear combination of the original descriptors) are supposed to maximally discriminate between the $k$ identified classes of the system. The other aspect of the discriminant analysis is the classification one. The purpose of the classification is principally to allocate a new observation to one of the $k$ identified classes of the system. In the remainder of the article, the Discriminant Analysis (DA) will be viewed only as a supervised classification method (Duda et al., 2001).

DA is based on the Bayes decision rule. This rule allocates a new observation $x$ to the class $C_{i}$ with the maximum a posteriori probability $p\left(C_{i} \mid x\right)$ giving the value of each descriptor, as defined in the equation 5.

$$
x \in C_{i}, \text { if } i=\underset{i=1, \ldots, k}{\operatorname{argmax}}\left\{p\left(C_{i} \mid x\right)\right\}
$$

This decision rule is named "Bayes decision rule" because it is based on the Bayes rule which gives the value of $p\left(C_{i} \mid x\right)$ as stated in 6.

$$
P\left(C_{i} \mid x\right)=\frac{p\left(C_{i}\right) p\left(x \mid C_{i}\right)}{p(x)}
$$

where $p\left(C_{i}\right)$ is the marginal probability of belonging to the class $C_{i}$. We can see that for each class, the denominator of the equation 6 is the same, so, it is not implicated in the discriminant function. Then, equation 5 can be rewritten as:

$$
x \in C_{i}, \text { if } i=\underset{i=1, \ldots, k}{\operatorname{argmax}}\left\{p\left(C_{i}\right) p\left(x \mid C_{i}\right)\right\}
$$

In industrial systems, it is usual to assume that data follow multivariate normal distribution. The density function conditionally to a class $C_{i}$ can be written as in equation 8 , where $\mu_{i}$ is the mean vector of the class $C_{i}, \Sigma_{i}$ is the covariance matrix of the class $C_{i}$ and $\left|\Sigma_{i}\right|$ represents the determinant of the matrix $\Sigma_{i}$.

$$
\phi\left(x \mid C_{i}\right)=\frac{\exp \left(-\frac{1}{2}\left(x-\mu_{i}\right)^{t} \Sigma_{i}^{-1}\left(x-\mu_{i}\right)\right)}{(2 \pi)^{p / 2}\left|\Sigma_{i}\right|^{1 / 2}}
$$

We remind that, for $n$ samples, the Maximum Likelihood Estimation (MLE) gives (Duda et al., 2001):

$$
\hat{\mu}=\frac{1}{n} \sum_{i=1}^{n} x_{i}
$$

and:

$$
\hat{\Sigma}=\frac{1}{n-1} \sum_{i=1}^{n}\left(x_{i}-\hat{\mu}\right)\left(x_{i}-\hat{\mu}\right)^{t}
$$

So, if our system has $k$ classes, the different probabilities can be computed with equation 11 .

$$
p\left(x \mid C_{i}\right)=\frac{\phi\left(x \mid C_{i}\right)}{\sum_{j=1}^{k} p\left(C_{j}\right) \phi\left(x \mid C_{j}\right)}
$$
12

So, the equation 7 can be rewritten by the equation

$$
x \in C_{i}, \text { if } i=\underset{i=1, \ldots, k}{\operatorname{argmax}}\left\{\frac{p\left(C_{i}\right) \phi\left(x \mid C_{i}\right)}{\sum_{j=1}^{k} \phi\left(x \mid C_{j}\right)}\right\}
$$

At the first view, we can tell that the DA is well suited for the fault diagnosis of industrial systems. But, we will show that we can also realize the fault detection methods of the multivariate control charts with a DA, and particularly with a BN. So, to do that we will consider the multivariate control charts as a DA where the goal is to attribute a class (in or out of control) to a new observation of the system. Realising a DA with a $\mathrm{BN}$ is very simple. The structure of the network will be composed of a class node (discrete variable) with two modalities (in or out of control). In order to represent the different descriptors of the system, we use only one normal multivariate variable. This normal multivariate variable will represent the $x$ for the $T^{2}$ chart and the $y_{t}$ for the MEWMA chart. To resume, we will obtain a structure similar to the CSNBN

However, an essential question we remain concerning the parameters of the network. We have to fix a normal multivariate distribution for each classes of the problem. The distribution of the "in control" state will be $N(\mu, \Sigma)$. In order to obtain a decision boundary between the two states of the process, we fix the distribution of the "out of control" state to $N(\mu, c \times \Sigma)$, where $c$ is a coefficient different than 1 . These two distributions will have same center $(\mu)$ and same shape (because $c \times \Sigma$ is simply a scale extending of the shape of $\Sigma$ ). The difference between the two states can be expressed as: scattering about the "out of control" state (OC) is more important than scattering about the "in control" state (IC). We can represent 


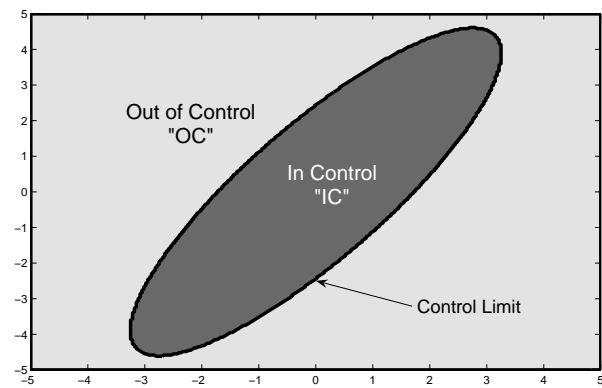

Figure 2: Example of the classification areas.

the classification areas of the two states of the system for a bivariate example like in the figure 2.

Usually, in the frame of Statistical Process Control (SPC) techniques, one has to choose a risk factor $\alpha$ in order to correctly control the process. In the case of the BN, we have to fix a probability threshold $\tau$ allowing to not badly reject some situations "in control" (false alarms).

Otherwise the threshold $\tau$ is computed in order to have similar classification areas than those of the multivariate control chart. We will name $C L$ the control limit for the control charts and we supposed that $C L$ is known for each chart, see (Montgomery, 1997; Lowry et al., 1992). The $\tau$ probability limit corresponds to the probability, for the class "OC", of the $x_{C L}$ value which is an observation giving a $T^{2}$ equal at the control limit $C L$. We will assume that the marginal probabilities of the two classes are unknown and so are fixed to 0.5 each. So, $p(I C)=p(O C)=0.5$.

$$
\begin{aligned}
\tau & =p\left(I C \mid x_{C L}\right) \\
& =\frac{p(O C) \phi\left(x_{C L} \mid O C\right)}{p(I C) \phi\left(x_{C L} \mid I C\right)+p(O C) \phi\left(x_{C L} \mid O C\right)} \\
& =\frac{\phi\left(x_{C L} \mid O C\right)}{\phi\left(x_{C L} \mid I C\right)+\phi\left(x_{C L} \mid O C\right)}
\end{aligned}
$$

By using equation 8 in the previous equation, and after simplification, we will obtain the equation of $\tau$ as:

$$
\tau=\frac{\frac{\exp \left(-0.5 \frac{C L}{c}\right)}{c^{p / 2}}}{\exp (-0.5 C L)+\frac{\exp \left(-0.5 \frac{C L}{c}\right)}{c^{p / 2}}}
$$

In conclusion, for the $\mathrm{BN}$, we will obtain the following decision rule: if $p(O C \mid x) \geq \tau$, then the process is out-of-control (OC), otherwise the process is in control (IC).

In order to use the previous result, we have taken a simple example of a 5 variables system. The parameters of the system is given as:

$$
\begin{aligned}
\mu & =[510] \\
\Sigma & =\left(\begin{array}{cc}
1 & 1.2 \\
1.2 & 2
\end{array}\right)
\end{aligned}
$$

We have simulated the system during 30 observations, but at the observation number 6 , a step of magnitude 0.5 is introduced in the process. In the figure 3 we are presenting the detection of the fault with the control chart (upper graph) and with the BN approach (lower graph). In a similar way, the figure 4 represents the result of the MEWMA approach.
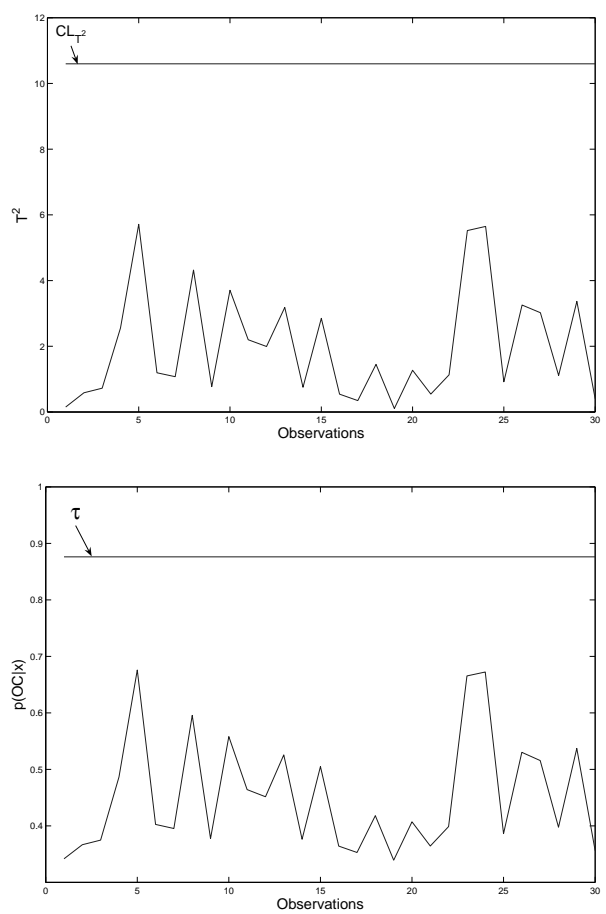

Figure 3: Results with the $T^{2}$ chart and the $T^{2}$ chart by BN apporach.

On the figure 3 and 4 , we can see that for each multivariate charts, the $\mathrm{BN}$ approach gives the same results than the specified chart (with a scaling factor). So, we can conclude that the different multivariate control charts ( $T^{2}$ and MEWMA) can be easily modeled with a BN.

\section{CONCLUSIONS AND OUTLOOKS}

In this article, after an introduction on the process control (and particularly on the data-driven techniques for the detection and the diagnosis), we have 

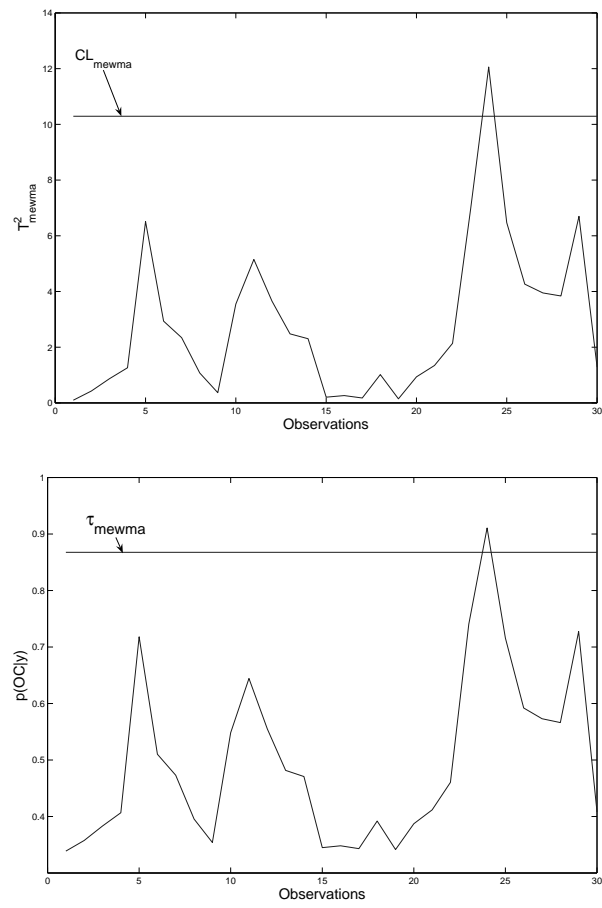

Figure 4: Results with the MEWMA chart and the MEWMA chart by BN approach.

outlined the problem that there is not a single tool able to perform both: the fault detection and the fault diagnosis of a system. As a bayesian network can be an efficient way to diagnosis a fault, it is natural to see how this tool can also execute the fault detection task. We have selected two fault detection techniques: the $T^{2}$ chart and the MEWMA chart. We have demonstrated that these charts can be viewed as a discriminant analysis and so can be implemented in a simple bayesian network.

The evident outlook to this work is the full study of the use of $\mathrm{BN}$ in order to monitor and control a multivariate process.

\section{REFERENCES}

Bakshi, B. R. (1998). Multiscale PCA with application to multivariate statistical process monitoring. AIChE Journal, 44(7):1596-1610.

Bodden, K. and Rigdon, S. (1999). A program for approximating the in-control arl for the mewma chart. Journal of Quality Technology, 31(1):120-123.

Chiang, L. H., Russell, E. L., and Braatz, R. D. (2001). Fault detection and diagnosis in industrial systems. New York: Springer-Verlag.

Cover, T. and Hart, P. (1967). Nearest neighbor pattern clas- sification. IEEE Transactions on Information Theory, 13:21-27.

Duda, R. O., Hart, P. E., and Stork, D. G. (2001). Pattern Classification 2nd edition. Wiley.

Friedman, N., Geiger, D., and Goldszmidt, M. (1997). Bayesian network classifiers. Machine Learning, 29(2-3):131-163.

Hotelling, H. (1947). Multivariate quality control. Techniques of Statistical Analysis, :111-184.

Jackson, E. J. (1985). Multivariate quality control. Communication Statistics - Theory and Methods, 14:2657 -2688 .

Jensen, F. V. (1996). An introduction to Bayesian Networks. Taylor and Francis, London, United Kingdom.

Kano, M., Nagao, K., Hasebe, S., Hashimoto, I., Ohno, H., Strauss, R., and Bakshi, B. (2002). Comparison of multivariate statistical process monitoring methods with applications to the eastman challenge problem. Computers and Chemical Engineering, 26(2):161174.

Kononenko, I. (1991). Semi-naive bayesian classifier. In EWSL-91: Proceedings of the European working session on learning on Machine learning, pages 206219.

Kourti, T. and MacGregor, J. F. (1996). Multivariate spc methods for process and product monitoring. Journal of Quality Technology, 28(4):409-428.

Langley, P., Iba, W., and Thompson, K. (1992). An analysis of bayesian classifiers. In National Conference on Artificial Intelligence.

Lowry, C. A., Woodall, W. H., Champ, C. W., and Rigdon, S. E. (1992). A multivariate exponentially weighted moving average control chart. Technometrics, 34(1):46-53.

MacGregor, J. and Kourti, T. (1995). Statistical process control of multivariate processes. Control Engineering Practice, 3(3):403-414.

Montgomery, D. C. (1997). Introduction to Statistical Quality Control, Third Edition. John Wiley and Sons.

Pearl, J. (1988). Probabilistic Reasoning in Intelligent Systems: Networks of Plausible Inference. Morgan Kaufmann Publishers.

Perez, A., Larranaga, P., and Inza, I. (2006). Supervised classification with conditional gaussian networks: Increasing the structure complexity from naive bayes. International Journal of Approximate Reasoning, 43:1-25.

Pignatiello, J. and Runger, G. (1990). Comparisons of multivariate cusum charts. Journal of Quality Technology, 22(3):173-186.

Shewhart, W. A. (1931). Economic control of quality of manufactured product. New York: D. Van Nostrand Co.

Vapnik, V. N. (1995). The Nature of Statistical Learning Theory. Springer. 